\title{
Adiposity and dyslipidaemia are associated with epigenetic age acceleration
}

\section{Abstract}

Adipose tissue is an endocrine organ involved in a variety of regulatory functions beyond simple fat storage. Excessive fat accumulation in the visceral tissue has been related to obesity associated comorbidities and manifestations such as hypertension, hyperglycaemia, hypercholesterolemia, and inflammatory processes. In the later stages of life, there is a shift of fat distribution from subcutaneous to visceral depots, which is associated to the development of several age-related diseases. Epigenetics has been described as a potential contributor in aging processes, being also associated with diseases and fat deposition that progress with age. The aim of this research was to investigate the relationships between aging, epigenetic processes and visceral adipose tissue.

The study population included 269 adult subjects recruited in the University of Navarra, Spain. Methylation data was assessed by Infinium MethylationEPIC beadchip from Illumina. Epigenetic age acceleration was calculated using the method GrimAge (AgeAccGrim), available in the website DNA methylation Age Calculator (https://dnamage.genetics.ucla.edu/home). Anthropometric, biochemical and blood pressure measurements were assessed following standardized methods. Body composition measurements by DXA were also carried out.

Statistically significant correlations were found between age acceleration and waist circumference, some DXA-measured variables (lean mass, trunk fat mass, android fat mass, visceral adipose tissue mass), glucose, HDL-cholesterol, triglycerides levels and C-reactive protein. Linear regression models showed that visceral adipose tissue mass and HDL-cholesterol were conjointly influencing the epigenetic age acceleration. In addition, a mediation by HDL-cholesterol in the relationship between AgeAccGrim and visceral adipose tissue mass was found.

Collectively, these findings demonstrated that visceral adiposity and dyslipidaemia are associated with accelerated aging effects, contributing to understand the development of age-related diseases.

\section{Conflict of Interest}

There is no conflict of interest 